INPLASY

PROTOCOL

To cite: Zheng et al.

Rehabilitation effect of lowerlimb motor function in stroke patients with Chinese traditional exercises : A systematic review and Metaanalysis. Inplasy protocol 202220039. doi:

10.37766/inplasy2022.2.0039

Received: 13 February 2022

Published: 13 February 2022

Corresponding author:

Yun Xiang

littlexiang20000@foxmail.com

Author Affiliation:

Chengdu Sport University

Support: National Natural

Science Found.

Review Stage at time of this submission: Completed but not published.

Conflicts of interest:

None declared.

\section{Rehabilitation effect of lower-limb motor function in stroke patients with Chinese traditional exercises: A systematic review and Meta-analysis}

Zheng, $\mathrm{Y}^{1} ; \mathrm{Xiang} \mathrm{Y}^{2}$.

Review question / Objective: To systematically evaluate the effectiveness of Chinese traditional exercises (CTE) in the rehabilitation of lower-limb motor function in stroke patients by Meta analysis statistical method.

Condition being studied: Stroke and motor dysfunction.

Main outcome(s): Fugl-Meyer Motor Scale (FMA) and Berg Balance Scale (BBS), the higher the score, the lower the degree of Motor dysfunction; The timed "Up \& Go" test shows that the shorter the test time is, the lower the degree of motor dysfunction.

INPLASY registration number: This protocol was registered with the International Platform of Registered Systematic Review and Meta-Analysis Protocols (INPLASY) on 13 February 2022 and was last updated on 13 February 2022 (registration number INPLASY202220039).

\section{INTRODUCTION}

Review question / Objective: To systematically evaluate the effectiveness of Chinese traditional exercises (CTE) in the rehabilitation of lower-limb motor function in stroke patients by Meta analysis statistical method.
Rationale: The CNKI, CBM, Wanfang, VIP, PubMed, Embase and Cochrane databases were searched by computer. The collection time was from the establishment of the database to May 2020. The subjects of the study were stroke patients and the intervention measures were randomized controlled trials of Chinese traditional exercises. Two researchers independently screened the literature, evaluated the quality of the literature, and extracted the 
data. RevMan5.3 software was used for Meta-analysis of the study.

Condition being studied: Stroke and motor dysfunction.

\section{METHODS}

Search strategy: Seven databases including CNKI, CBM, Wanfang, VIP, PubMed, Embase and Cochrane were searched in this study. The time limit for collection is from the establishment of the database to May 2020. The Participants of the study were stroke patients and the intervention was a randomized controlled trial using Chinese traditional exercises. The retrieval method combines subject words with free words. English search terms mainly included Stroke, Strokes, Cerebrovascular Accident, Tai Ji, Baduanjin, Qigong, randomized, etc. Chinese search terms include stroke, apoplexy, taijiquan, yi jin jing, Ba duan jin, guidance, qigong, wuqinxi, randomized control, etc.

Participant or population: The patient's age, gender and case origin were not limited. The first diagnosis was stroke and motor dysfunction. Stroke diagnosis of cerebral apoplexy by the world health organization, the definition accord with the Chinese medical association branch of neurology cardiovascular epidemiology group cerebrovascular disease diagnosis main point of the "China 2019" diagnostic criteria[15], and confirmed by CT or MRI brain stroke patients.

Intervention: The intervention measures were traditional Chinese exercises (Tai Chi, Ba Duan Jin, Wuqinxi, Gui Ying, Yi Jin Jing) or combined with other treatments.

Comparator: Conventional rehabilitation treatment.

Study designs to be included: Randomized controlled trial.

Eligibility criteria: Participants The patient's age, gender and case origin were not limited. The first diagnosis was stroke and motor dysfunction. Stroke diagnosis of cerebral apoplexy by the world health organization, the definition accord with the Chinese medical association branch of neurology cardiovascular epidemiology group cerebrovascular disease diagnosis main point of the "China 2019" diagnostic criteria[15], and confirmed by CT or MRI brain stroke patients.Intervention Control group: conventional rehabilitation treatment; Experiment group: The intervention measures were traditional Chinese exercises (Tai Chi, Ba Duan Jin, Wuqinxi, Gui Ying, Yi Jin Jing) or combined with other treatments. Study type Randomized controlled trial Outcome FuglMeyer Motor Scale (FMA) and Berg Balance Scale (BBS), the higher the score, the lower the degree of Motor dysfunction; The timed "Up \& Go" test shows that the shorter the test time is, the lower the degree of motor dysfunction. Exclusion criteria Excluded literatures with no available data or incomplete data, literatures with repeated publication, literatures without full text access, literatures with unreasonable research design or poor quality, reviews and conference articles.

Information sources: Electronic databases.

Main outcome(s): Fugl-Meyer Motor Scale (FMA) and Berg Balance Scale (BBS), the higher the score, the lower the degree of Motor dysfunction; The timed "Up \& Go" test shows that the shorter the test time is, the lower the degree of motor dysfunction.

Data management: Two researchers independently screened the literature, assessed the literature quality and extracted the data. If there is a disagreement between the two researchers, a third party will be consulted to assist in the judgment. If there is any missing information, try to contact the author and make up for it.Manage literature with NoteExpress.The included studies were used for data synthesis, and RevMan5.3 was used for data analysis.

Quality assessment / Risk of bias analysis: In accordance with evidence-based medicine research guidelines, 2 
researchers independently used the Cochrane bias risk assessment tool to assess the quality of the included studies in six aspects [15]: including the generation of random sequence, distribution, the implementation of the hidden method, blind method, the result data integrity and selective reports the results of the study, and other sources of bias.

Strategy of data synthesis: The included studies were used for data synthesis, and RevMan5.3 was used for data analysis. FMA, BBS and TUGT were all measurement data. In this meta-analysis, point estimates and $95 \% \mathrm{Cl}$ were given for each effect size, and mean difference (MD) was used as the effect index. When heterogeneity (12 50\%), the random-effect model was used for data analysis. $P$ is used to evaluate whether the results have statistical significance. When $\mathrm{P} 0.05$, were considered not statistically significant. In addition, if there is significant heterogeneity, subgroup analysis or sensitivity analysis will be used to deal with it, and descriptive analysis will be used for studies that cannot carry out data synthesis.

Subgroup analysis: 2756 patients in 39 studies were included in this study, with a total of . According to different outcome indicators, traditional Chinese exercises were divided into Taijiquan group, Baduanjin group, Daoyin group, Yijinjing group and Wuqinxi group for subgroup analysis. MD was taken as the effect indicator, and used random effect model for analysis 1.FMA and FMA lower limbs; 2.BBS; 3.TUGT.

Sensitivity analysis: After subgroup analysis, when there is still significant heterogeneity between studies, sensitivity analysis is required, that is, Meta-analysis of the remaining studies and whether the results change, so as to assess whether the Meta-analysis results are stable and reliable.

Language: Chinese and English literature.
Keywords: Traditional Chinese exercises; Rehabilitation; Stroke; Meta-analysis.

Contributions of each author:

Author 1 - ying zheng - The first author is responsible for literature retrieval, data collection, literature quality assessment, data analysis and article writing.

Email: 1012806326@qq.com

Author 2 - yun xiang - The corresponding author is responsible for literature quality assessment, data analysis and review, and ensuring the authenticity of the paper.

Email: littlexiang20000@foxmail.com

Country(ies) involved: China. 